J. Urol. Urogynäkol. AT 2020 • 27:41-42 https://doi.org/10.1007/s41972-019-00090-6 Online publiziert: 8. Januar 2020

(c) Der/die Autor(en) 2020

\section{Barbara Bodner-Adler}

Abteilung für Allgemeine Gynäkologie und Gynäkologische Onkologie, Universitätsfrauenklinik, Medizinische Universität Wien, Wien, Österreich

\title{
Perioperatives Kathetermanagement und Restharnmessungen in der Urogynäkologie
}

\section{Begriffsdefinition}

Der Begriff perioperativ umfasst den Zeitraum

- vor (präoperativ),

- während (intraoperativ) und

- nach (postoperativ) einer Operation.

Präoperativ ist die Restharnmessung (RH: Restharn) ein wesentlicher Teil in der Urogynäkologie, welche regelmäßig im Rahmen der Basisdiagnostik durchgeführt wird. Das Kathetermanagement umfasst intraoperativ am Ende einer Inkontinenz- oder Prolapsoperation meist das Setzen ein Dauerkatheters (DK), welcher üblicherweise am nächsten Tag in der Früh entfernt werden kann.

\section{Postoperatives Harnverhalten}

Postoperatives Harnverhalten ist ein häufiges Problem nach Inkontinenzoder Prolapsoperationen. Durch ödematöse und entzündliche Gewebeveränderungen sowie Schmerzen kann es zu einer unvollständige Blasenentleerung kommen (mit daraus resultierender inadäquater RH-Bildung). Die Inzidenz des postoperativen Harnverhaltens liegt zwischen 1,4 und $40 \%$, wobei die große Schwankungsbreite dadurch erklärt wird, dass keine Standarddefinition bezüglich signifikanter RH-Menge vorhanden ist. Klinisch äußert sich ein postoperatives Harnverhalten durch eine unvollständige Blasenentleerung mit RH-Bildung.

Literatur beim Verfasser
Dieses kann unmittelbar postoperativ oder verzögert auftreten, partiell oder komplett sein, symptomatisch oder asymptomatisch sowie vorübergehend oder persistierend.

\section{Risikofaktoren}

Als Risikofaktoren für ein postoperatives Harnverhalten gelten ein Alter $>50$ Jahre, weibliches Geschlecht, fortgeschrittener Beckenorganprolaps (BOP), primäre Blasendysfunktion, vorausgegangene Inkontinenzoperation, gynäkologische oder kolorektale chirurgische Eingriffe, intraoperative Flüssigkeitsgabe $>750 \mathrm{ml}$, Opioidgebrauch sowie das Vorhandensein eines postoperativen HWI.

\section{Folgen}

Bei rechtzeitiger Diagnosestellung ergeben sich keine wesentlichen Konsequenzen. Bei verzögerter Diagnosestellung entstehen durch die verlängerte Blasenüberdehnung einerseits Symptome („lower urinary tract symptoms“ [LUTS]), andererseits auch eine negative Beeinflussung des korrigierten OP-Gebietes. Bei fehlender Diagnose kann es zu ernsthaften klinischen Folgen durch die verlängerte Blasenüberdehnung kommen.

Die frühzeitige Erkennung ist somit essenziell, um eine Blasenüberdehnung zu vermeiden und die Möglichkeit der adäquaten und rechtzeitigen Harnableitung zu nützen. Der Schlüssel zur rich- tigen Therapie liegt somit in der Früherkennung.

\section{Früherkennung}

Die Bestimmung der RH-Menge sollte prinzipiell nach jeder Prolapsoperation (v. a. im Bereich des vorderen Kompartiments) oder Inkontinenz-OP durchgeführt werden. Die Definition einer inadäquaten RH-Menge ist allerdings nicht einheitlich.

Als RH wird allgemein "die in der Blase verbleibende Harnmenge am Ende oder nach einer normalen Miktion" bezeichnet, wobei die Grenzwerte schwach und uneinheitlich definiert sind. Als allgemeine Richtwerte gelten:

- $\mathrm{RH}<50 \mathrm{ml}$ : normal,

- RH zwischen 50 und $100 \mathrm{ml}$ : Untergrenze einer inadäquaten $\mathrm{RH}$ Menge,

- RH zwischen 200 und $300 \mathrm{ml}$ : inadäquat; Symptome und Auswirkungen auf postoperatives Ergebnis.

Die RH-Messung erfolgt entweder mittels Einmalkatheter (EK) oder mittels Ultraschall und Berechnung des Blasenvolumens (transabdominal: Blasenvolumen in $\mathrm{ml}=\mathrm{H} \times \mathrm{W} \times \mathrm{D} \times 0,7)$.

\section{RH-Messung}

Bei allen Patientinnen, welche eine Behandlung erhalten haben, die potenziell eine Blasenentleerungsstörung verursachen kann, sollte eine RH-Messung durchgeführt werden (LOE 1b; Empfeh- 
lungsgrad B), das bedeutet nach einer Inkontinenzoperation oder nach einer Prolapsoperation (vorderes Kompartiment).

\section{Behandlungsstrategien}

In der Literatur existiert keine Evidenz bezüglich des Kathetermanagements bei inadäquaten RH-Mengen nach einer Prolapsoperation. Das Risiko eines unnötig langen Katheterismus sowie das Risiko für eine höhere Rate an HWI erhöhen sich dadurch.

Eine multizentrische randomisiert kontrollierte Studie (RCT) verglich beispielsweise intermittierenden Selbstkatheterismus (ISK) mit einem Dauerkatheter (DK) über 3 Tage bei Patientinnen mit inadäquaten $\mathrm{RH}-$ Mengen $(>150 \mathrm{ml})$ nach einer Prolaps-OP. Es zeigte sich ein signifikant geringeres Risiko für eine Bakteriurie sowie für einen HWI in der ISK-Gruppe $(p<0,05)$. Weiters war die Dauer der Behandlung deutlich geringer in der ISK-Gruppe, und eine Normalisierung der RH-Mengen trat $54 \mathrm{~h}$ früher auf als in der DK-Gruppe $(p<0,05)$. Bezüglich Schmerzen und Zufriedenheit war kein Unterschied zwischen den beiden Kathetergruppen zu verzeichnen. Als Schlussfolgerung dieser Arbeit ergab sich, dass der ISK als eine bessere Therapieoption nach einer Prolaps-OP mit vorübergehender $\mathrm{RH}$-Bildung gilt.

\section{Zusammenfassung und Empfehlung}

- Das Intervall zwischen Blasenentleerung und RH-Messung sollte möglichst kurz gehalten werden.

- Es existiert keine allgemein akzeptierte Definition einer signifikanten RH-Menge, wobei allerdings RHMengen $<30 \mathrm{ml}$ als klinisch normal gelten.

- Hohe RH-Mengen (>200-300 ml) können LUTS indizieren und das OP-Ergebnis negativ beeinflussen (EL 3).

- Die Aufklärung über die Möglichkeit einer vorübergehenden postoperativen Blasenentleerungsstörung nach einem urogynäkologischen Eingriff erscheint essenziell und beinhaltet auch die Aufklärung bezüglich ISK.
- Obwohl die Rate an persistierenden Blasenentleerungsstörungen generell gering bleibt, kann durch eine adäquate präoperative Aufklärung eine bessere Akzeptanz der Patientin erreicht werden.

- Ziele: Studien mit größerer Patientenanzahl und besserem Studiendesign sowie eine Unterscheidung zwischen Hoch- und Niedrigrisikokollektiven für postoperative Blasenentleerungsstörung wären sinnvoll und wünschenswert.

\section{Korrespondenzadresse

Assoc.-Prof. Univ.-Doz. Dr.
Barbara Bodner-Adler
Abteilung für Allgemeine
Gynäkologie und
Gynäkologische Onkologie,
Universitätsfrauenklinik,
Medizinische Universität
Wien
Währinger Gürtel 18-20,
1090 Wien, Österreich
barbara.bodner-adler@
meduniwien.ac.at

Funding. Open access funding provided by Medical University of Vienna.

\section{Einhaltung ethischer Richtlinien}

Interessenkonflikt. B. Bodner-Adler gibt an, dass kein Interessenkonflikt besteht.

Für diesen Beitrag wurden von der Autorin keine Studien an Menschen oder Tieren durchgeführt. Für die aufgeführten Studien gelten die jeweils dort angegebenen ethischen Richtlinien.

Weitere Details zur Lizenz entnehmen Sie bitte der Lizenzinformation auf http://creativecommons.org/ licenses/by/4.0/deed.de.

Die in diesem Artikel enthaltenen Bilder und sonstiges Drittmaterial unterliegen ebenfalls der genannten Creative Commons Lizenz, sofern sich aus der Abbildungslegende nichts anderes ergibt. Sofern das betreffende Material nicht unter der genannten Creative Commons Lizenz steht und die betreffende Handlung nicht nach gesetzlichen Vorschriften erlaubt ist, ist für die oben aufgeführten Weiterverwendungen des Materials die Einwilligung des jeweiligen Rechteinhabers einzuholen.

Open Access. Dieser Artikel wird unter der Creative Commons Namensnennung 4.0 International Lizenz veröffentlicht, welche die Nutzung, Vervielfältigung Bearbeitung, Verbreitung und Wiedergabe in jeglichem Medium und Format erlaubt, sofern Sie den/die ursprünglichen Autor(en) und die Quelle ordnungsgemäß nennen, einen Link zur Creative Commons Lizenz beifügen und angeben, ob Änderungen vorgenommen wurden.
Hinweis des Verlags. Der Verlag bleibt in Hinblick auf geografische Zuordnungen und Gebietsbezeichnungen in veröffentlichten Karten und Institutsadressen neutral. 